\title{
Design of a Novel UWB Omnidirectional Antenna Using Particle Swarm Optimization
}

\author{
Chengyang $\mathrm{Yu}$, Tanghong $\mathrm{Xu}$, and Changjun Liu \\ School of Electronics and Information Engineering, Sichuan University, Chengdu 610064, China \\ Correspondence should be addressed to Changjun Liu; cjliu@ieee.org
}

Received 23 January 2015; Revised 11 March 2015; Accepted 13 March 2015

Academic Editor: Stefano Selleri

Copyright (c) 2015 Chengyang Yu et al. This is an open access article distributed under the Creative Commons Attribution License, which permits unrestricted use, distribution, and reproduction in any medium, provided the original work is properly cited.

\begin{abstract}
A UWB E-plane omnidirectional microwave antenna is designed and fabricated for IEEE 802.11a communication system and microwave magnetron source system as a radiation monitor. A cooptimization method based on particle swarm optimization (PSO) algorithm and FDTD software is presented. The presented PSO algorithm is useful in many industrial microwave applications, such as microwave magnetron design and other techniques with a high power level. The maximum measured relative bandwidth of $65 \%$ is achieved for the proposed antenna after a rapid and efficient optimization. Furthermore, the measured antenna polarization purity reaches about $20 \mathrm{~dB}$ at the communication $\mathrm{C}$ band. The PSO algorithm is a powerful candidate for microwave passive component design.
\end{abstract}

\section{Introduction}

Omnidirectional antennas are widely used in wireless communication systems, especially for high-multipath communication applications based on polarization diversity technique. A typical polarization diversity system is composed of two orthogonally polarized antennas, such as a vertically polarized monopole and a horizontally polarized Alford loop antenna. As an $\mathrm{H}$-plane omnidirectional antenna, monopole has been widely researched. However, in such a situation, Eplane omnidirectional antennas are also needed to investigate. Alford loop antenna, which is suitable at low frequencies with the wire type, was firstly reported in [1]. Several improved antennas based on Alford structure were also investigated to generate E-plane omnidirectional radiation patterns [25]. In [3], a dual-frequency Alford structure loop antenna is realized with eight T-dipoles. However, broadband omnidirectional antennas are urgently needed for modern communication systems $[6,7]$.

In this paper, an ultrawideband (UWB) characteristic is realized on the Alford structure loop antenna with Eplane omnidirectionality. Such an antenna will be used as a radiation monitor at an actual microwave magnetron source system. Particle swarm optimization (PSO) algorithm is introduced to optimize the whole structure. The proposed antenna can be easily realized on a planar substrate while it has a far-field radiation pattern similar to that of a magnetic dipole. In addition, the optimized omnidirectional antenna has a measured impedance bandwidth from 4.6 to $9.0 \mathrm{GHz}$ (relative bandwidth is about 65\%), which covers the entire $5 \mathrm{GHz}$ bandwidth of IEEE $802.11 \mathrm{a}(5.15 \mathrm{GHz}-5.35 \mathrm{GHz}$ and $5.725-5.875 \mathrm{GHz})$.

\section{Antenna Design}

2.1. Antenna Structure. The configuration of the proposed UWB omnidirectional antenna is shown in Figure 1. It mainly consists of three identical pairs of printed half-wave dipole radiators. Each pair includes two dipoles which work together to generate a broadband characteristic. It is the distributed microstrip dipoles and the power combining structure that generate an omnidirectional feature for the antenna. The lengths of the two dipole radiators are $2 R_{1} \times \theta_{1}$ and $2 R_{2} \times$ $\theta_{2}$, respectively. Combining with double-sided strip lines, two parts of a dipole radiator are fabricated on the opposite sides of one substrate. So the proposed dipole structure is 


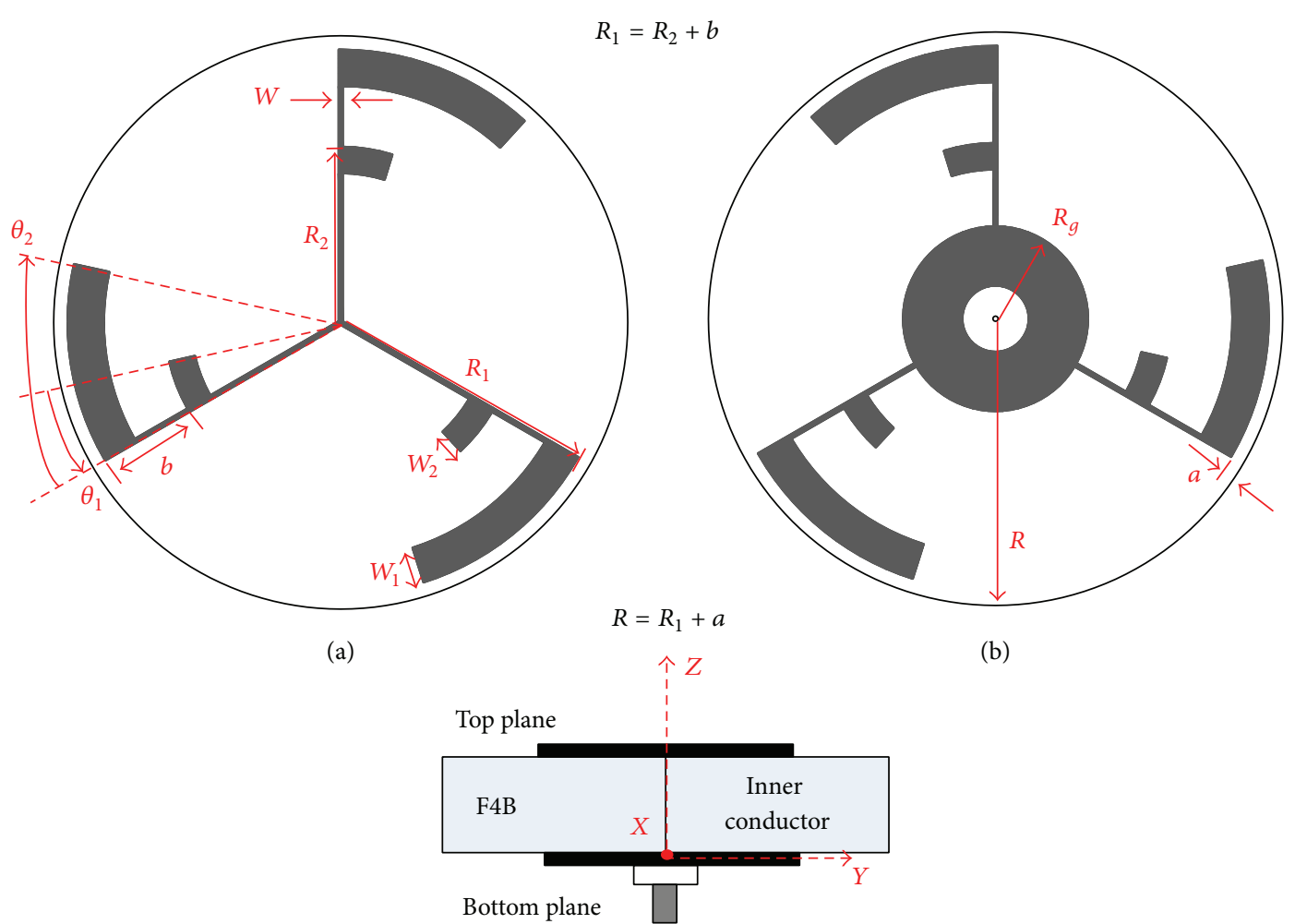

(c)

FIGURE 1: The structure of the microwave antenna: (a) top plane, (b) bottom plane, and (c) side view.

TABLE 1: Range setup of optimized parameters.

\begin{tabular}{lccccccccccc}
\hline Parameters & $W_{1}$ & $W_{2}$ & $W$ & $\theta_{1}$ & $\theta_{2}$ & $a$ & $b$ & $R_{2}$ & $R_{1}$ & $R$ & $R_{g}$ \\
Optimization range & $0.1 \mathrm{~mm}-4 \mathrm{~mm}$ & $\pi / 18-\pi / 3$ & $0.1 \mathrm{~mm}-2 \mathrm{~mm}$ & $0.1 \mathrm{~mm}-6 \mathrm{~mm}$ & $8 \mathrm{~mm}-14 \mathrm{~mm}$ & $R_{2}+b$ & $R_{1}+a$ & $6 \mathrm{~mm}$ \\
Restricted condition & - & - & - & - & - & - & - & $R_{2}<R_{1}<R$ & Constant \\
\hline
\end{tabular}

equivalent to the conventional dipole. In order to form an omnidirectional radiation, according to the theory of antenna array, the excitation phase of each dipole pair should be equal. The three dipole pairs are directly fed by microstrips, while the common ground plane of microstrips is a circular patch with a constant radius $R_{g}$. The center of the ring structure is soldered with an SMA connector. It is obvious that all the dipole pairs are fed with not only an equal excitation phase, but also an equal excitation amplitude.

2.2. Antenna Optimization and Fitness Function. Based on the proposed structure, the final goal of our work is to obtain a planar antenna with omnidirectional radiation and low return loss over WLAN operation in the $5 \mathrm{GHz}$ bands. However, due to the narrow impedance bandwidth of conventional dipole, massive optimizations on radiators and connection structures among them are needed. In order to improve optimization accuracy and velocity, a cooptimization method based on PSO and FDTD simulator is introduced in this paper. The PSO algorithm and cooptimization processes will be detailed later.

The omnidirectional antenna is realized on a F4B substrate with a dielectric constant of 2.65 and thickness of $1 \mathrm{~mm}$. The specific optimized parameters of the proposed antenna, as shown in Figure 1, are listed in Table 1. Parameters " $a$ " and " $b$ " are selected to match the restricted condition among kinds of radiuses of the antenna structure.

According to design targets of the proposed antenna, especially used on the entire $5 \mathrm{GHz}$ bandwidth of IEEE 802.11a, the fitness function can be defined as

$$
\text { Fitness }=0.5 \times \mathrm{BW}+A+B \text {, }
$$

where BW indicates the desired antenna impedance bandwidth expressed in terms of upper frequency $f_{U}$ and lower frequency $f_{L}$. The upper and lower frequencies are the boundary points of antenna bandwidth with $\mathrm{dB}\left(S_{11}\right)<$ $-10 \mathrm{~dB}$. $A$ and $B$ represent the weight factor of reflection 


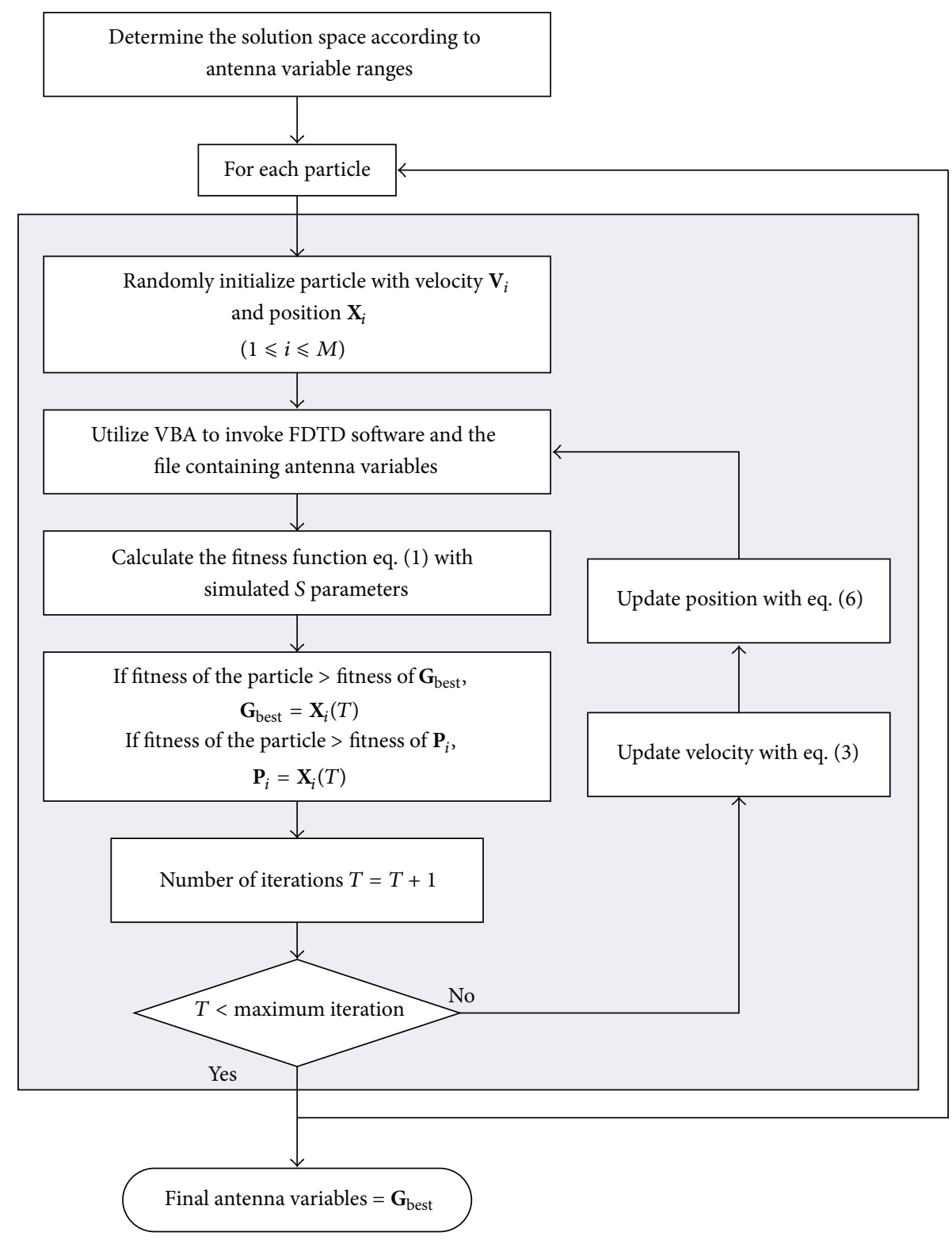

FIGURE 2: Flow chart of the proposed antenna cooptimization method.

coefficient to optimize on the antenna at $5.2 \mathrm{GHz}$ and $5.8 \mathrm{GHz}$, respectively. These factors can be expressed by

$$
\begin{gathered}
\mathrm{BW}= \begin{cases}\frac{f_{U}-f_{L}}{1 \mathrm{GHz}}, & f_{U}>6 \mathrm{GHz}, f_{L}<5 \mathrm{GHz} \\
0, & \text { others, }\end{cases} \\
A= \begin{cases}1, & \left.\mathrm{~dB}\left(S_{11}\right)\right|_{f=5.2 \mathrm{GHz}} \leq-10 \mathrm{~dB} \\
0, & \left.\mathrm{~dB}\left(S_{11}\right)\right|_{f=5.2 \mathrm{GHz}}>-10 \mathrm{~dB},\end{cases} \\
B= \begin{cases}1, & \left.\mathrm{~dB}\left(S_{11}\right)\right|_{f=5.8 \mathrm{GHz}} \leq-10 \mathrm{~dB} \\
0, & \left.\mathrm{~dB}\left(S_{11}\right)\right|_{f=5.8 \mathrm{GHz}}>-10 \mathrm{~dB} .\end{cases}
\end{gathered}
$$

2.3. PSO Algorithm and Cooptimization with FDTD Software. As an evolutionary computation technique based on the movement and intelligence of particle swarm, PSO is presented by Kennedy et al. [8]. Each particle in the swarm represents a possible solution to the specific optimization event. There are $M$ particles to search an $N$ dimensions solution space, respectively. So the velocity, position, and the personal best position are expressed by $M \times N$ matrixes. The position of particle $i$ at a fixed iteration $T$ is usually expressed as a vector $\mathbf{X}_{i}(T)=\left[X_{i, 1}(T), X_{i, 2}(T), \ldots, X_{i, N}(T)\right]$, where $i$ satisfies $1 \leq i \leq M$. This particle adjusts its position with velocity $\mathbf{V}_{i}(T)=\left[V_{i, 1}(T), V_{i, 2}(T), \ldots, V_{i, N}(T)\right]$ through the solution space. According to the fitness function calculation, the personal best particle and global best particle are involved in $\mathbf{P}_{i}=\left[P_{i, 1}, P_{i, 2}, \ldots, P_{i, N}\right]$ and $\mathbf{G}_{\text {best }}=\left[G_{1}, G_{2}, \ldots, G_{N}\right]$. 

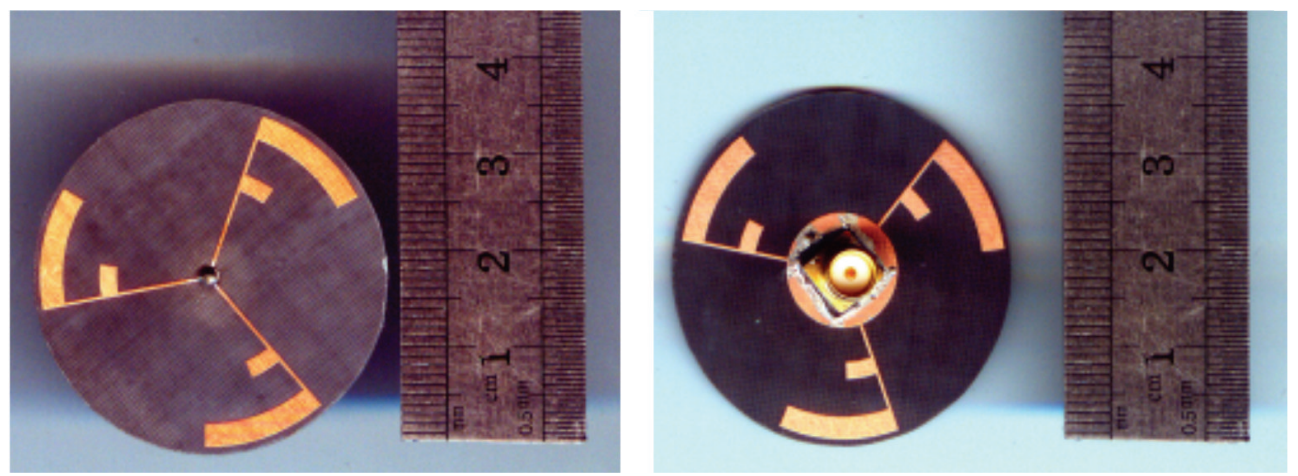

FIgURE 3: The fabricated UWB omnidirectional microwave antenna (top view and bottom view).

Clerc and Kennedy have introduced a constriction factor [9], $K$, which is used to constrain and control velocities for PSO. In [10], Eberhart and Shi concluded that the PSO using a constriction factor $K$ is the best approach while limiting the maximum velocity $V_{\max }$ to the dynamic range of variable $X_{\max }$ on each dimension compared with performance using an inertia weight. The velocity function of PSO used in this paper is

$$
\begin{aligned}
V_{i, j}(T+1)=K \times[ & V_{i, j}(T)+\phi_{1} \operatorname{rand}() \times\left(P_{i, j}(T)-X_{i, j}(T)\right) \\
+ & \left.\phi_{2} \operatorname{rand}() \times\left(G_{j}(T)-X_{i, j}(T)\right)\right],
\end{aligned}
$$

where the constriction factor $K$ is computed as

$$
\begin{array}{r}
K=\frac{2}{\left|2-\phi-\sqrt{\phi^{2}-4 \phi}\right|} \\
\phi=\phi_{1}+\phi_{2}>4 .
\end{array}
$$

We tested different groups of the cognitive and social component values of the PSO $\left(\phi_{1}\right.$ and $\left.\phi_{2}\right)$ with Griewank function and Sphere function. The standard value settings in [10] $\left(\phi_{1}=\phi_{2}=2.05\right)$ and those in [11] $\left(\phi_{1}=2.8\right.$ and $\left.\phi_{2}=1.3\right)$ result in a better optimization accuracy and a better convergence rate, respectively. In this paper, aiming at a compromise on performances, improved cognitive and social component values are used for the PSO. Cognitive and social rates vary from 2.8 to 2.05 and from 1.3 to 2.05 , synchronously. The variation is linear to iteration times. Tested results show that the proposed settings of $\left(\phi_{1}=2.8 \sim 2.05\right.$ and $\left.\phi_{2}=1.3 \sim 2.05\right)$ result in the best performance on optimization accuracy and a good convergence rate for PSO.

Reflecting boundary condition [12] is used to limit the particle velocity and position when it hits the boundary in one of the dimensions. The particle velocity and position beyond the boundary can be expressed by

$$
\begin{gathered}
V_{i, j}(T+1)=-m_{j} V_{i, j}(T), \\
X_{i, j}(T+1)=X_{i, j}(T)+V_{i, j}(T+1),
\end{gathered}
$$

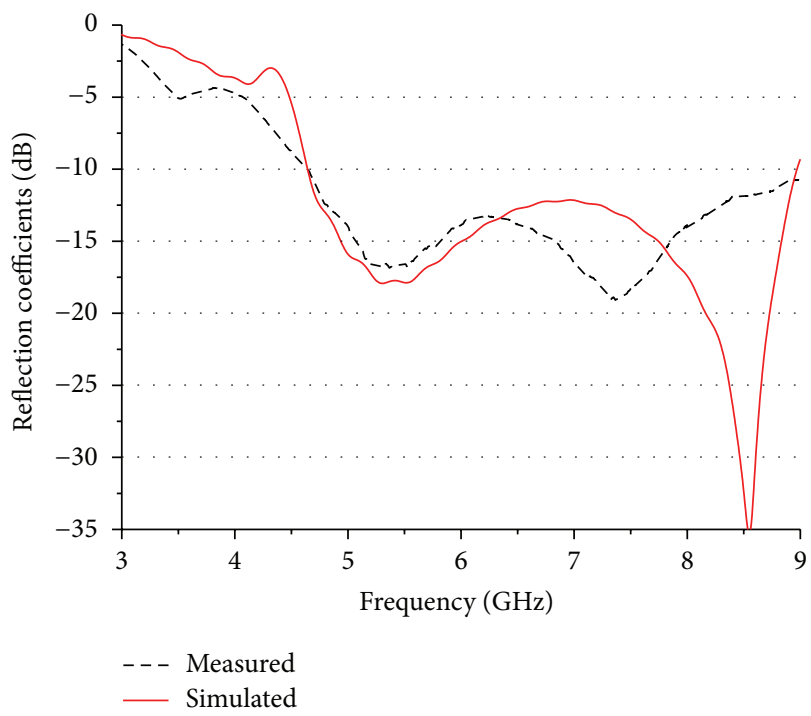

FIGURE 4: Simulated and measured results of reflection coefficient.

where $m_{j}$ is determined by the distance $d$ from particle position to the boundary:

$$
m_{j}= \begin{cases}\frac{d}{X_{j}^{\max }-X_{j}^{\min }}, & d \leq X_{j}^{\max }-X_{j}^{\min } \\ \frac{X_{j}^{\max }-X_{j}^{\min }}{d} & d>X_{j}^{\max }-X_{j}^{\min } .\end{cases}
$$

The steps of cooptimization with the proposed PSO and FDTD software (CST) are described in Figure 2.

Step 1. Determine the antenna variables and ranges to be optimized. Randomly initialize $M$ particles with velocity $\mathbf{V}_{i}$ and position $\mathbf{X}_{i}$ in the solution space.

Step 2. Write the variables into a.txt file at fixed position. Invoke the file and CST software automatically by using VBA (a macro language of Visual Basic). Use the simulated $S$ parameters to calculate the fitness of each particle according to (1). Record the personal particles and global best particle according to the fitness function value. 

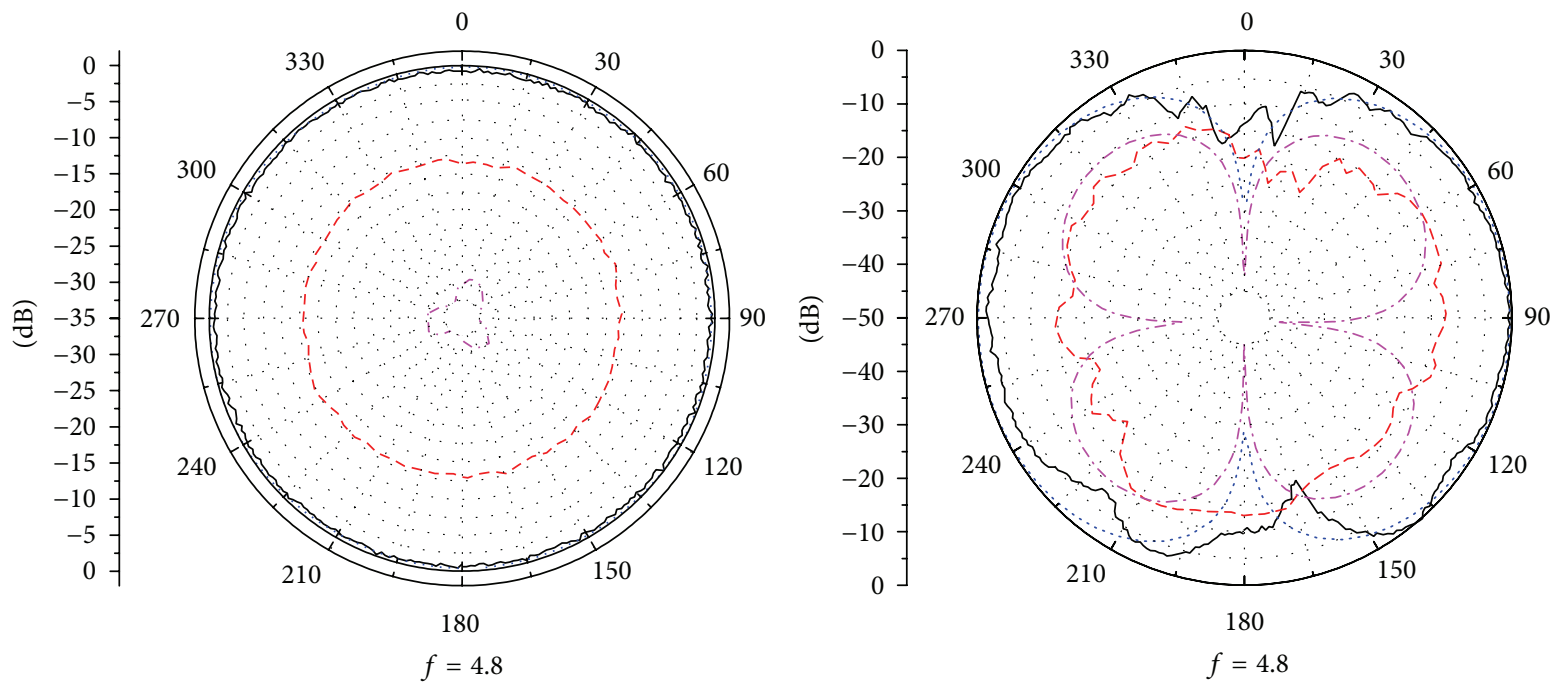

(a) At $4.8 \mathrm{GHz}$

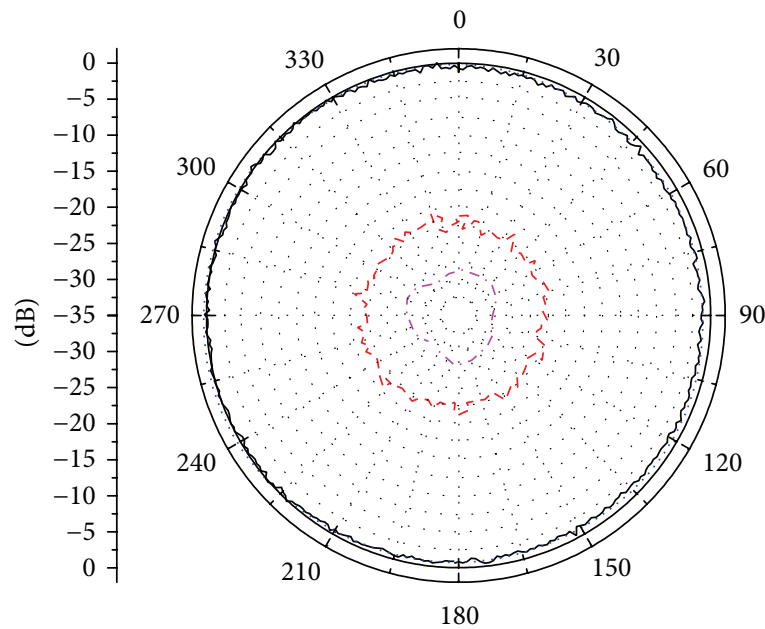

$f=5.2$

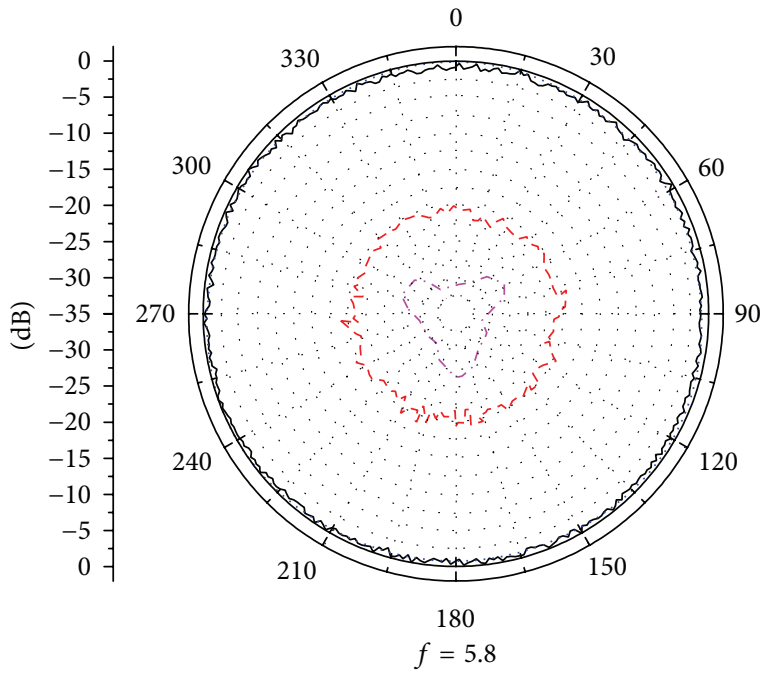

$\begin{array}{ll}\text { - } & \text { Measured E } \\ --- & \text { Measured Ex }\end{array}$

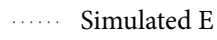

...- Simulated Ex

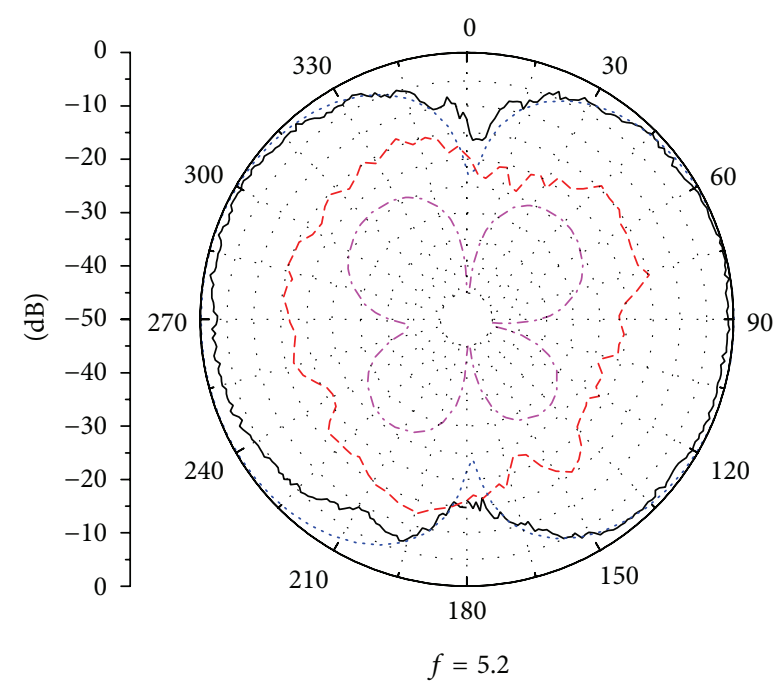

(b) At $5.2 \mathrm{GHz}$

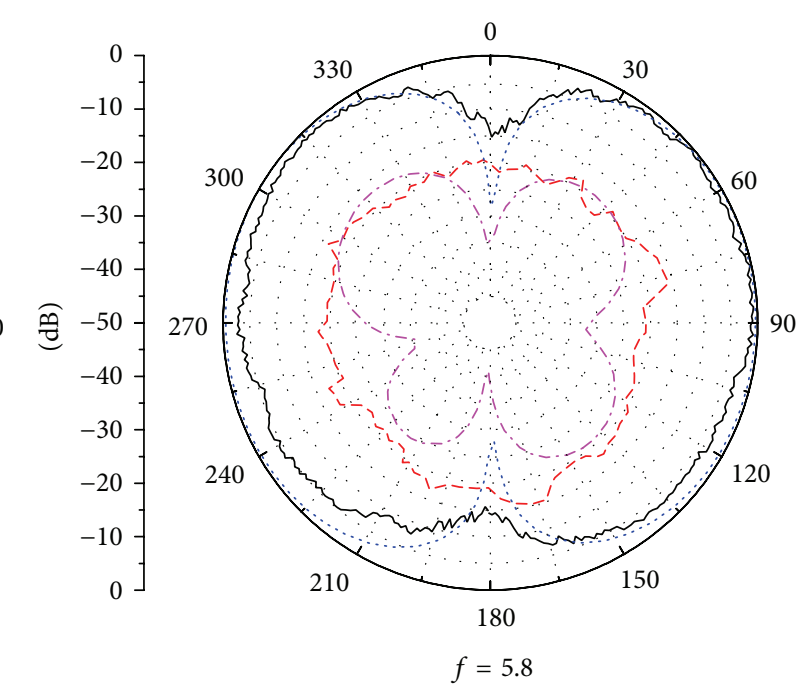

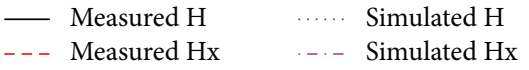

(c) At $5.8 \mathrm{GHz}$

FIgure 5: Continued. 

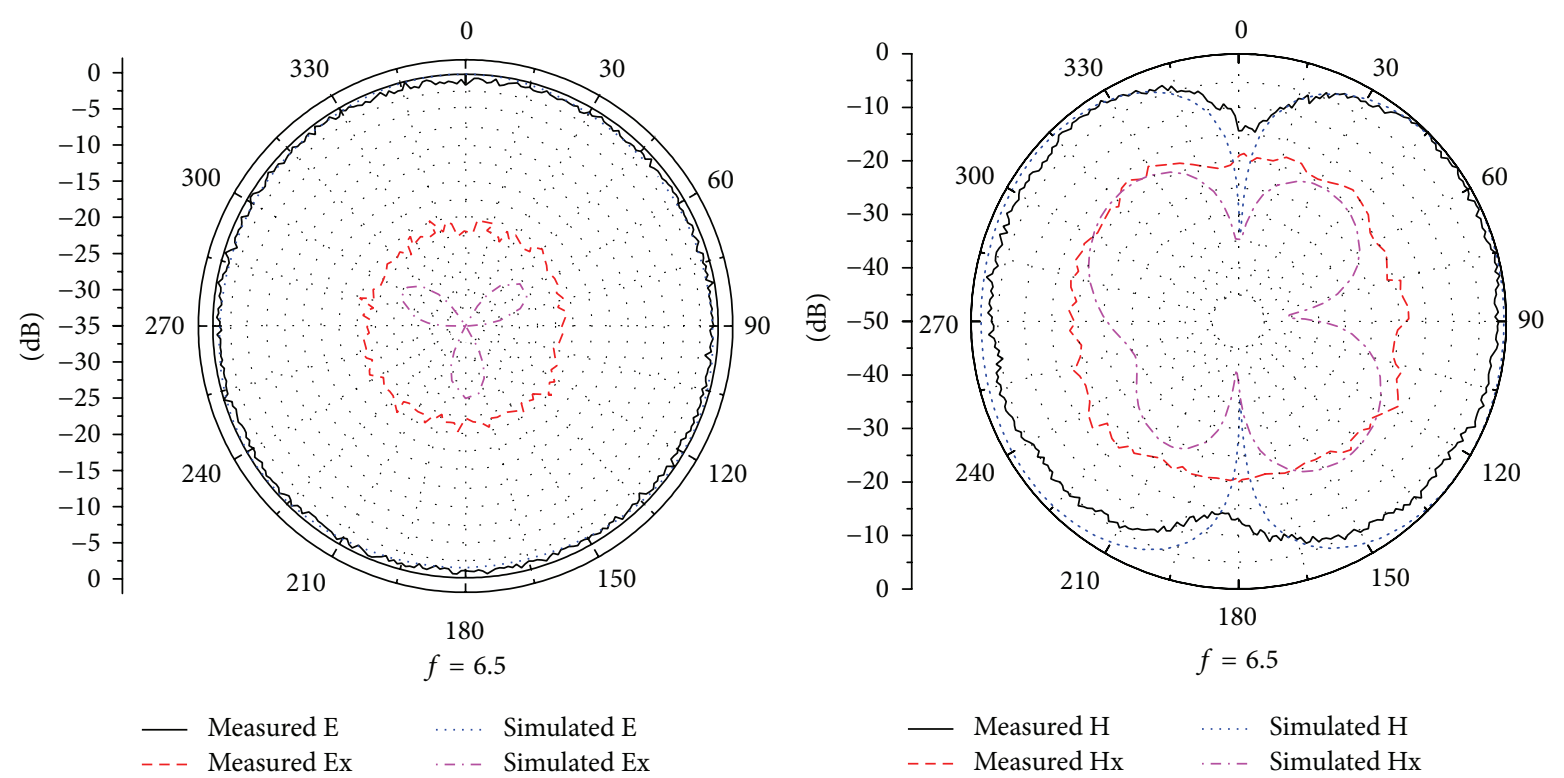

(d) At $6.5 \mathrm{GHz}$

FIGURE 5: Simulated and measured microwave radiation patterns at (a) $4.8 \mathrm{GHz}$, (b) $5.2 \mathrm{GHz}$, (c) $5.8 \mathrm{GHz}$, and (d) $6.5 \mathrm{GHz}$.

Step 3. Update the velocity and position of each particle according to (3) and (6).

Step 4. Calculate the fitness of each particle again. Update the personal particles.

Step 5. Read the personal best particle. If its corresponding fitness function value is better than that of global best particle, update the record of the global best particle.

Step 6. Repeat Steps 3, 4, and 5, until the maximum iteration number is reached.

\section{Fabrication and Measurements}

The omnidirectional antenna mentioned above is optimized with the proposed optimization method based on PSO and FDTD software. The operation frequency of this antenna is the entire $5 \mathrm{GHz}$ wideband of IEEE 802.11a, especially for frequency bands of $5.2 \mathrm{GHz}$ and $5.8 \mathrm{GHz}$. For obtaining a fine UWB characteristic, 20 particles and 150 iteration times are employed. The final optimized geometric parameters are $a=0.88 \mathrm{~mm}, b=3.90 \mathrm{~mm}, R_{2}=9.65 \mathrm{~mm}, \theta_{1}=0.30$, $\theta_{2}=0.74, W=0.30 \mathrm{~mm}, W_{1}=2.39 \mathrm{~mm}$, and $W_{2}=1.75 \mathrm{~mm}$. Figure 3 shows the fabricated UWB omnidirectional antenna. The diameter of this antenna is $36 \mathrm{~mm}$.

The reflection coefficient was measured using an Agilent N5230A vector network analyzer. As shown in Figure 4, the simulated results matched well with the measured results. This indicates that the cooptimization method based on PSO and FDTD software is effective for antenna design. Furthermore, the improved Alford structure antenna, without any additional matching circuits, definitely has an UWB characteristic from $4.6 \mathrm{GHz}$ to $9.0 \mathrm{GHz}$.
The radiation patterns of the proposed antenna are measured and simulated at $4.8 \mathrm{GHz}, 5.2 \mathrm{GHz}, 5.8 \mathrm{GHz}$, and $6.5 \mathrm{GHz}$. Figure 5 shows the comparison of simulated and measured patterns which include the coplanar polarization ( $\mathrm{E}$ and $\mathrm{H}$ ) and cross polarization (Ex and $\mathrm{Hx}$ ) of the antenna. It is obvious that the proposed antenna has an excellent omnidirectional radiation in the entire $5 \mathrm{GHz}$ band of IEEE 802.11a. The measured polarization purity in the E-plane reaches about $20 \mathrm{~dB}$. The data differences between measured and simulated polarization purity parameters are mainly caused by the noise background of power receiver in the antenna measurement system. However, $20 \mathrm{~dB}$ polarization purity is good enough to be an E-plane omnidirectional antenna of polarization diversity system.

The simulated and measured antenna gains are shown in Figure 6. The maximum measured antenna gains are 1.3 and $1.0 \mathrm{dBi}$ at 5.2 and $5.8 \mathrm{GHz}$, respectively.

\section{Conclusion}

A novel UWB E-plane omnidirectional antenna has been proposed for polarization diversity of IEEE 802.11a communication system and some industrial applications. Power combining construction with three microstrip dipoles is investigated to form the omnidirectional radiation feature. The PSO algorithm is a powerful candidate for the design and optimization on the proposed UWB antenna. The measured results show that the antenna has a relative bandwidth of $65 \%$ ( 4.6 to $9.0 \mathrm{GHz}$ ). The good measured omnidirectional radiation feature in the $5 \mathrm{GHz}$ band enables the antenna to operate at IEEE 802.11a system and monitor the radiation level in microwave magnetron source effectively. Furthermore, it is experimentally demonstrated that the proposed 


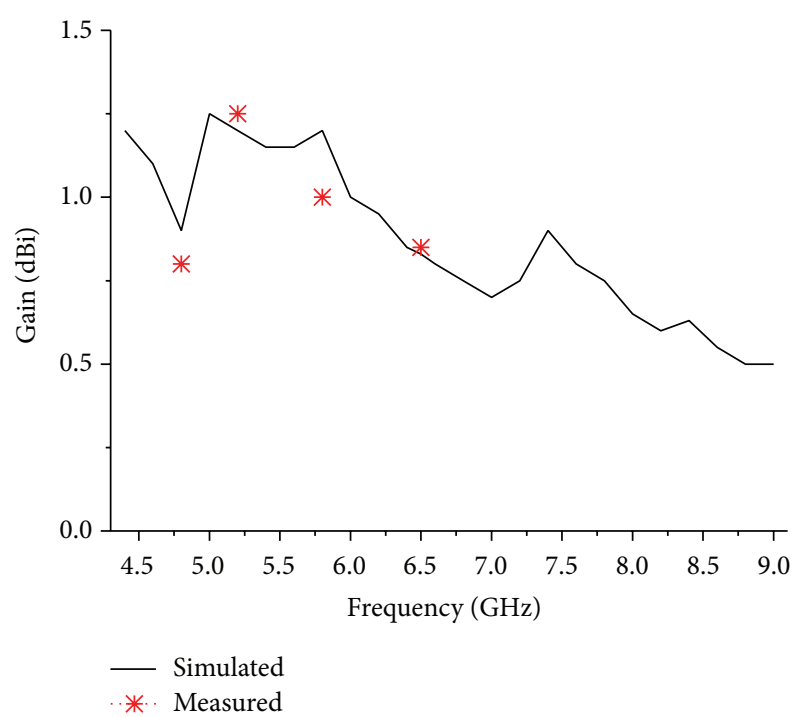

FIGURE 6: Simulated and measured results of the microwave antenna gain.

E-plane omnidirectional antenna is suitable for realizing polarization diversity technique associated with an $\mathrm{H}$-plane omnidirectional antenna.

\section{Conflict of Interests}

The authors declare that there is no conflict of interests regarding the publication of this paper.

\section{Acknowledgments}

This work was supported in part by the 973 Program 2013CB328902, NSFC 0971051, and NCET-12-0383.

\section{References}

[1] A. Alford and A. G. Kandoian, "Ultra-high frequency loop antenna," AIEE Transactions, vol. 59, no. 12, pp. 843-848, 1940.

[2] A. J. Fenn, "Arrays of horizontally polarized loop-fed slotted cylinder antennas," IEEE Transactions on Antennas and Propagation, vol. 33, no. 4, pp. 375-382, 1985.

[3] C.-H. Ahn, S.-W. Oh, and K. Chang, "A dual-frequency omnidirectional antenna for polarization diversity of MIMO and wireless communication applications," IEEE Antennas and Wireless Propagation Letters, vol. 8, pp. 966-969, 2009.

[4] C.-C. Lin, L.-C. Kuo, and H.-R. Chuang, "A horizontally polarized omnidirectional printed antenna for WLAN applications," IEEE Transactions on Antennas and Propagation, vol. 54, no. 11, pp. 3551-3556, 2006.

[5] H.-R. Chuang and L.-C. Kuo, "3-D FDTD design analysis of a 2.4-GHz polarization-diversity printed dipole antenna with integrated balun and polarization-switching circuit for WLAN and wireless communication applications," IEEE Transactions on Microwave Theory and Techniques, vol. 51, no. 2, pp. 374-381, 2003.

[6] T. Sedghi, M. Jalali, and T. Aribi, "Fabrication of CPW-fed fractal antenna for UWB applications with omni-directional patterns," The Scientific World Journal, vol. 2014, Article ID 391602, 5 pages, 2014.

[7] M. N. Iqbal, Hamood-Ur-Rahman, and S. F. Jilani, "An ultrawideband monopole fractal antenna with coplanar waveguide feed," International Journal of Antennas and Propagation, vol. 2014, Article ID 510913, 7 pages, 2014.

[8] J. F. Kennedy, R. Eberhart, and Y. Shi, Swarm Intelligence, Elsevier Science, 2001.

[9] M. Clerc and J. Kennedy, "The particle swarm-explosion, stability, and convergence in a multidimensional complex space," IEEE Transactions on Evolutionary Computation, vol. 6, no. 1, pp. 58-73, 2002.

[10] R. C. Eberhart and Y. Shi, "Comparing inertia weights and constriction factors in particle swarm optimization," in Proceedings of the 2000 Congress on Evolutionary Computation, vol. 1, pp. 84-88, July 2000.

[11] A. Carlisle and G. Dozier, "An off-the-shelf PSO," in Proceedings of the Workshop on Particle Swarm Optimization, Indianapolis, Ind, USA, 2001.

[12] J. Robinson and Y. Rahmat-Samii, "Particle swarm optimization in electromagnetics," IEEE Transactions on Antennas and Propagation, vol. 52, no. 2, pp. 397-407, 2004. 

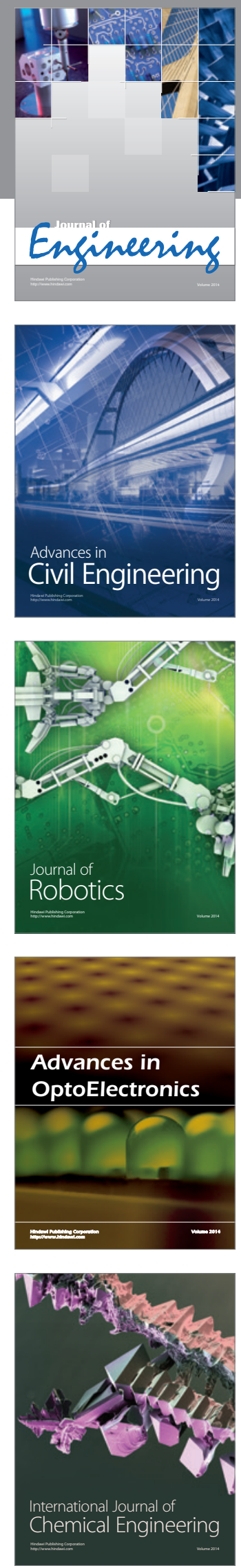

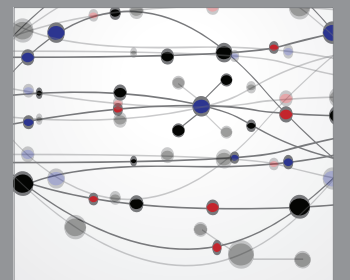

The Scientific World Journal
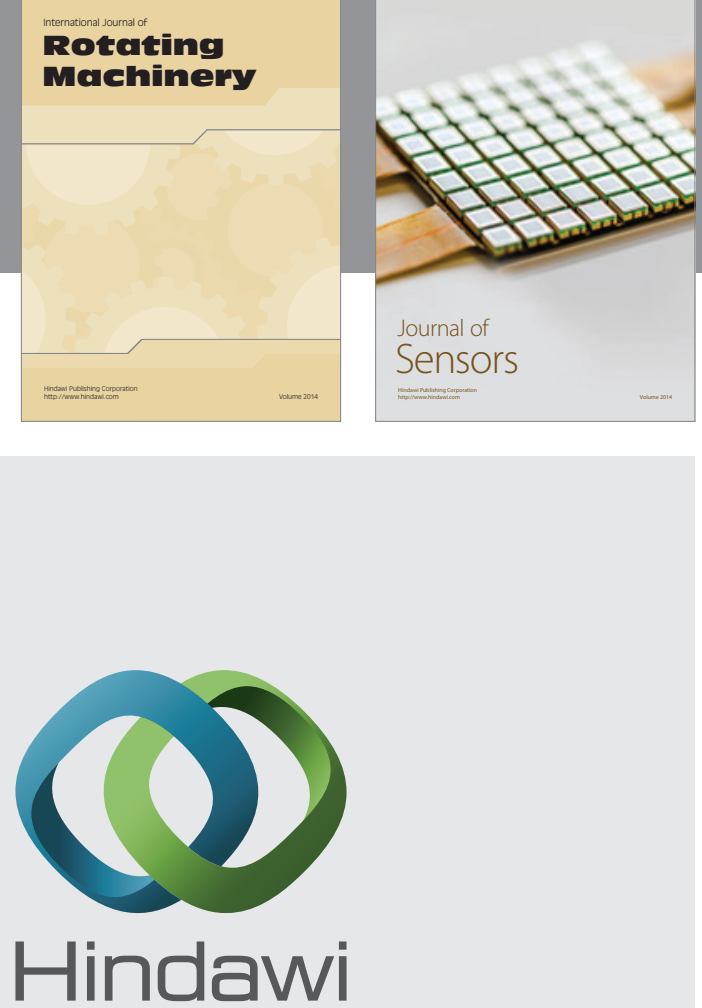

Submit your manuscripts at http://www.hindawi.com
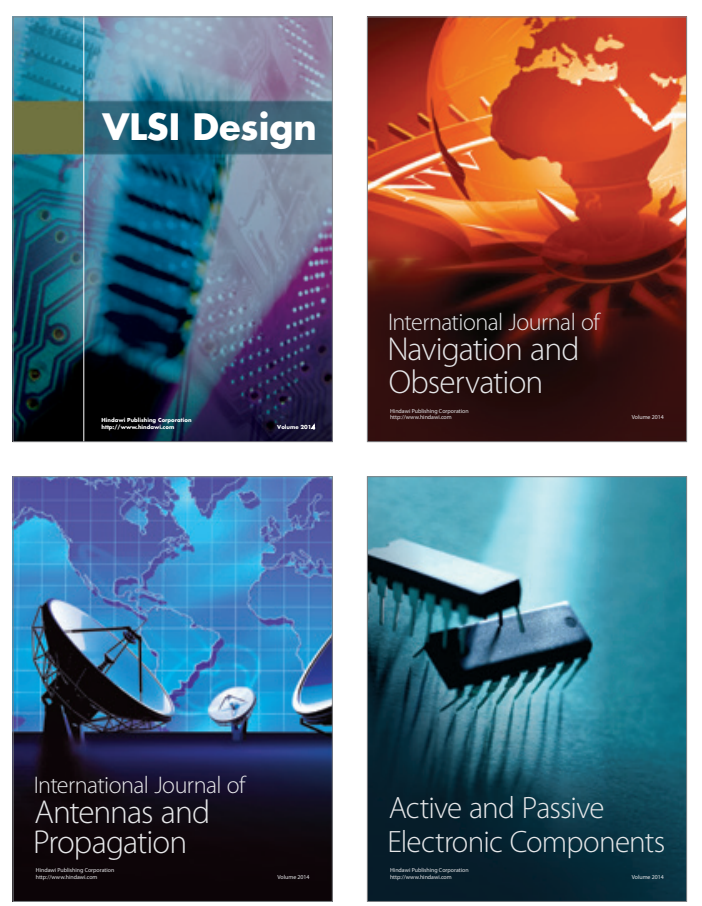
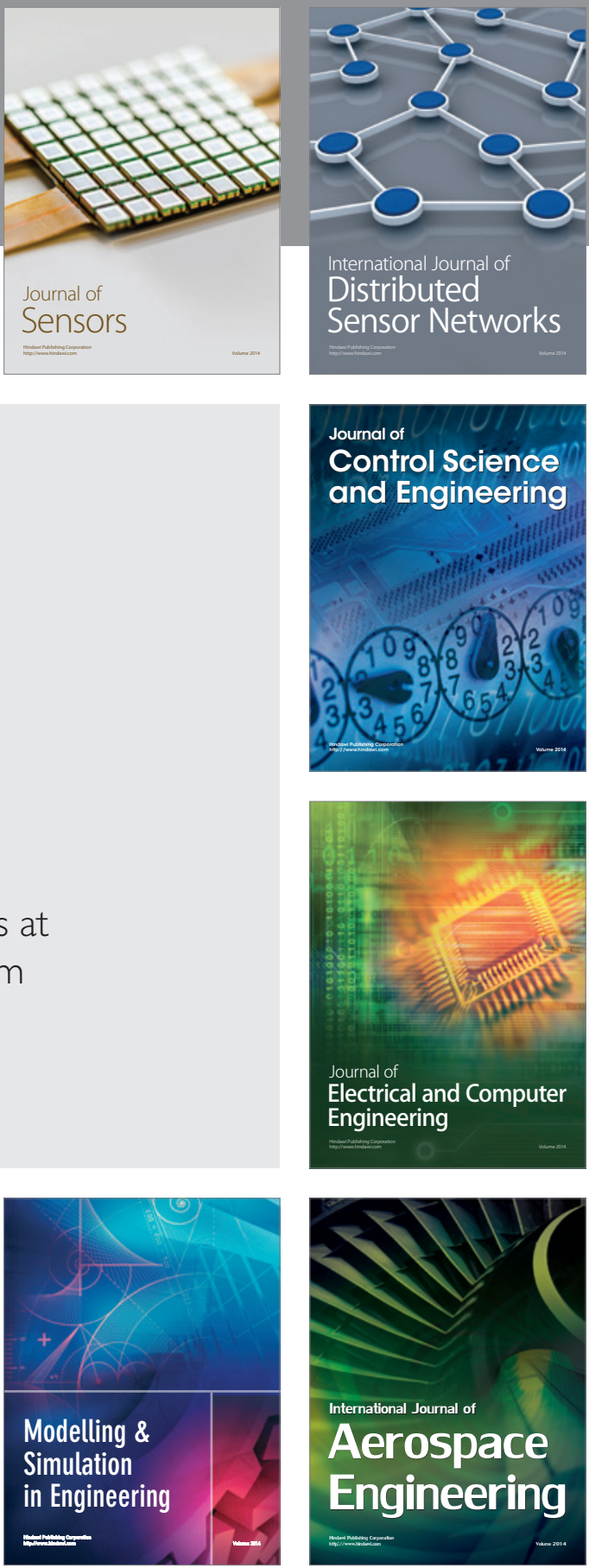

Journal of

Control Science

and Engineering
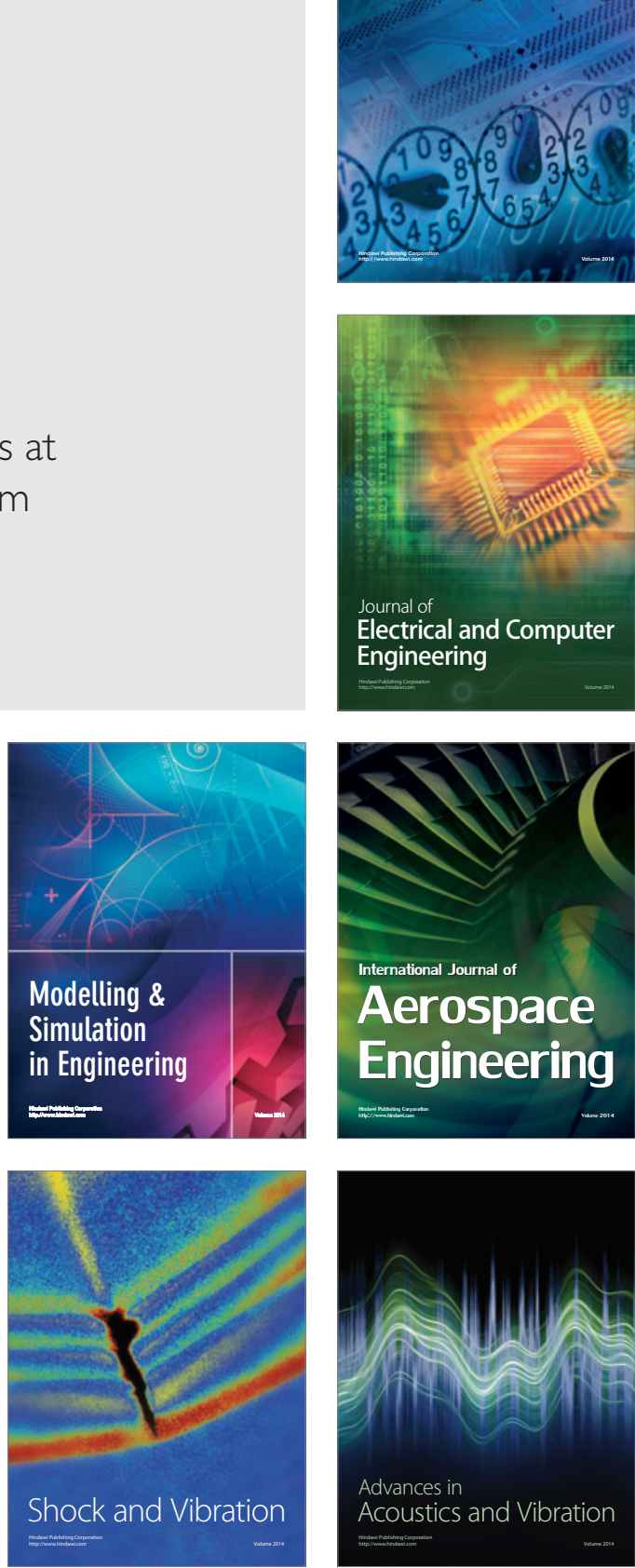